\title{
Low-Carb/High-Fat/Fried-Food Diet: The Surprising Effects in Triglycerides, Lipid Profile, and Glycemia in a Patient with a Metabolic Syndrome
}

\section{Baldini C}

Bionutrizione Organica - Nutrizione dello Sport, Italy

*Correspondence: Cristian Baldini, Bionutrizione Organica - Nutrizione dello Sport, Italy

Received on 28 July 2021; Accepted on 10 September 2021; Published on 15 September 2021

Copyright (C) 2021 Baldini C. This is an open access article and is distributed under the Creative Commons Attribution License, which permits unrestricted use, distribution, and reproduction in any medium, provided the original work is properly cited.

\begin{abstract}
We continuously underestimate the eating process: food and cooking methods play a pivotal role in our health. According to the Dietary Guidelines for Americans (DGA), more than 117 million American adults have one or more preventable chronic diseases, many of which are related to poor quality eating patterns and physical inactivity. Despite the usual belief about fat in the diet, it has shown that fat has a uniquely positive effect on blood lipid concentrations and cardiovascular risk factors. A low-carb/high-fat/fried-food (LCHFFF) diet has shown to be one of the best natural treatments in lipid profile and glycemia in a patient with metabolic syndrome.
\end{abstract}

Keywords: triglycerides, lipid, glycemia, metabolic syndrome, cardiovascular, cholesterol

Abbreviations: LCHFFF: low-carb/high-fat/fried-food; T2D: type 2 diabetes; LCHF: low in carb and high in fat; CVD: cardiovascular disease

\section{Introduction}

Generally, the word metabolic syndrome is referred to a group of risk factors that can lead to conditions such as diabetes, stroke, and other health problems. The five metabolic risk factors include a large waistline, high triglyceride level, low HDL cholesterol level, high fasting blood sugar, and high blood pressure. Most of the published reports indicate that the syndrome does not predict cardiovascular events or disease progression any better than the sum of its components $[1,2]$.

The relationship between a diet and health is extremely complex. Strategies to prevent or delay chronic diseases such as cardiovascular disease (CVD) are of utmost interest because chronic diseases and more concretely CVD are the leading cause of death and disability worldwide [3]. 
Despite of the fact that most of the countries dietary guidelines recommend that people with metabolic syndrome or type 2 diabetes (T2D) must limit their consumption of fats and cholesterol, a diet low in carb and high in fat (LCHF) has been shown to have a uniquely positive effect on the lipid profile and glycemia [4].

The higher incidence of CVD in egg consumers is more likely to be caused by the clustering of other CVD risk factors [3].

For example, a diet rich in eggs doesn't show any negative effect on blood lipid levels in T2D patients [5]. Highquality intervention studies have found non-significant effects of increasing the consumption of eggs on risk markers for CVD and T2D in healthy subjects and subjects with T2D [6]. Also, it is well-recognized that nuts, considerably high in fats, can lower blood cholesterol concentrations and modify blood lipoprotein levels [7].

Cooking methods also play a pivotal role in our health; nobody recommends a specific way to cook a specific food. First of all, a specific way that foods are cooked can change the therapeutical destiny of that meal. According to Farnetti et al. [8], in obese insulin-resistant women, food fried in extra-virgin olive oil significantly reduced both insulin and C-peptide responses after meal with respect to the same food when boiled.

Also, each meal (how we combine foods and different type of cooking methods) have a unique effect in our physioendocrine system.

The liver plays an interesting role in lipid metabolism; degrade fatty acids into small compounds that can be used for energy, synthesize triglycerides (mainly from carbohydrates), synthesize other lipids from fatty acids, especially cholesterol and phospholipids. Almost all the quantity of carbohydrates that enters the body is used immediately for energy (or can be stored in the form of glycogen), and the excess is rapidly converted into triglycerides and stored in this form in the adipose tissue [9-12].

The origin of cholesterol has two different sources, i.e., exogenous cholesterol, and an even greater quantity called endogenous cholesterol. Practically all the endogenous cholesterol that circulates in the lipoproteins of the plasma is formed by the liver, but all other cells of the body form at least some cholesterol. Cholesterol is the precursor of bile salt. About 1-2 $\mathrm{g}$ of cholesterol is removed from the blood plasma and secreted into the bile each day in the process of secreting the bile salts. Despite the dietary recommendation from almost all the national dietary associations, food rich in good fat, cholesterol, and fried food in olive oil has a strong positive effect on triglycerides level, lipid panel in general, and glycemia.

Blood tests after 1 and 2 months of this dietary treatment (LCHFFF diet) show how triglycerides decrease about 66\%. A positive effect was registered in the blood lipid profile (LDL, HDL, total cholesterol) and the control of glycemia [9-12].

\section{Objective}

The goal of this dietary intervention was to check if a diet with fried-fatty food can also improve triglycerides in patients with metabolic syndrome. However, a strong positive effect in glycemia, HbA1c, and lipid panel (HDL/LDL levels and cholesterol ratio) was well documented $[4,13]$.

\section{Materials and Methods}

The patient is a female who was 65 years old at the time of the visit with $72 \mathrm{~kg}$ of weight and had mild T2D and mild hypertension. She was prescribed a week's renewable dietary strategy. Thanks to the help of a chef, she was instructed on how to cook the recipes, which foods and which cooking method will have a positive effect on her problem.

Before the start of the diet, she was prescribed by the general practitioner with statins, omega 3 , and hypoglycemic drugs to reduce cholesterol and triglycerides and control glycemia. 


\section{Dietary interventions}

As described previously, it was a renewable, customized diet for a week [13]. A particular emphasis was there on cooking methods. As described by Farnetti et al. [8], the same meal fried or boiled has a completely different effect on glycemia. It was seen in other cases that different cooking methods can have positive or negative effects in completely different conditions like ulcerative colitis, for example.

The patient was assigned to a low-carb/high-fat/fried-food (LCHFFF) diet. Dairy products were excluded from the diet. The limit of egg consumption was not mentioned. The principles of the diet were: 1) increase the content fat from extra-virgin olive oil (EVOO) and other good fats, 2) only EVOO for frying, 3) prefer fatty fish, 4) prefer pork fat, for example in pasta (amatriciana), 5) eat only nuts between the meals and only if hungry, 6) do not cross the limit of 50 $\mathrm{g}$ of bread or pasta during the day for each meal, 7) chose fruit with a low glycemic index, and 8) drink only water.

\section{Primary outcomes}

Bodyweight, waist circumference, the recording of side effects, any medication changes, and a dietetic review was registered during the diet.

The primary outcome that we registered was a huge decrease in triglyceride levels. Also, a positive effect on cholesterol level and glycemia was registered. All other assessments were secondary outcomes. Blood samples were collected from the laboratory for triglycerides, fasting blood glucose, HbA1c, total cholesterol, HDL cholesterol, LDL cholesterol, blood count, thyroid function, and liver and renal function.

To confirm this decrease of triglycerides, the blood tests were repeated after 3 days in another laboratory.

\section{Secondary outcome}

After 1.5 months of treatment, it was possible to deduce that the glycemia is strongly decreased as the HbA1c trend shows. At the time of writing, it is necessary to wait for 2 months to have more realistic data for the HbAlc.

There was no significant difference in the HDL cholesterol, an improvement was seen in the total cholesterol and weight loss (fat loss) was registered.

\section{Changes in anthropometric measures and vital signs}

Reduction in waist circumference and blood pressure was observed, and better control on feeling of satiety.

\section{Results}

After only a month of LCHFFF diet, the results were a massive reduction in triglycerides, improved control of glycemia, massive reduction in total cholesterol level, greater satiety, and better weight management. As shown in the table, the level of triglycerides was higher before $(437 \mathrm{mg} / \mathrm{dl})$ as compared after the diet $(217 \mathrm{mg} / \mathrm{dl})$ and even lesser in the second lab $(149 \mathrm{mg} / \mathrm{dl}$ ) (Table 1). This confirms that almost all the quantity of carbohydrates that enters the body is used immediately for energy. In fact, the HbAlc estimation was decreased after the $\operatorname{diet}(6.6 \% \mathrm{or} 49 \mathrm{mmol} / \mathrm{mol}$ to $6.0 \%$ or $42 \mathrm{mmol} / \mathrm{mol}$ ).

The total cholesterol decreased $(216 \mathrm{mg} / \mathrm{dl}$ to $112 \mathrm{mg} / \mathrm{dl})$ after a month of LCHFFF diet, a trend confirmed by the second lab in accordance with Noakes et al. [4]. No side effect was relevant in the liver and pancreatic functions as seen in the blood tests (Supplementary Data 1, 2 and 3). 


\begin{tabular}{|l|l|l|l|}
\hline & $\mathbf{5 - 1 4 - 2 0 2 1}$ & $\mathbf{6 - 1 5 - 2 0 2 1}$ & $\mathbf{6 - 1 7 - 2 0 2 1}$ Second Lab \\
\hline Triglyceride & $437(\mathrm{mg} / \mathrm{dl})$ & $217(\mathrm{mg} / \mathrm{dl})$ & $149(\mathrm{mg} / \mathrm{dl})$ \\
\hline Total cholesterol & $216(\mathrm{mg} / \mathrm{dl})$ & $112(\mathrm{mg} / \mathrm{dl})$ & $130(\mathrm{mg} / \mathrm{dl})$ \\
\hline LDL & & & $67(\mathrm{mg} / \mathrm{dl})$ \\
\hline HDL & $34(\mathrm{mg} / \mathrm{dl})$ & $36(\mathrm{mg} / \mathrm{dl})$ & $33(\mathrm{mg} / \mathrm{dl})$ \\
\hline HbA1c & $6.6 \%, 49 \mathrm{mmol} / \mathrm{mol} \mathrm{IFCC}$ & $6 \%, 42 \mathrm{mmol} / \mathrm{mol} \mathrm{IFCC}$ & \\
\hline AST/GOT & & & $16 \mathrm{U} / \mathrm{I}$ \\
\hline ALT/GPT & & & $26 \mathrm{U} / \mathrm{I}$ \\
\hline GGT & & & $25 \mathrm{U} / \mathrm{I}$ \\
\hline Alpha amilase & & & $20 \mathrm{U} / \mathrm{I}$ \\
\hline Lipase & & & $12 \mathrm{U} / \mathrm{I}$ \\
\hline LDH & & $214 \mathrm{U} / \mathrm{I}$ \\
\hline
\end{tabular}

Table 1: Results after a month of low-carb/high-fat/fried-food (LCHFFF) diet.

\section{Discussion}

Metabolic syndrome is a cluster of biological factors characterized by abdominal obesity, dyslipidemia, hypertension, and T2D conditions where the diet plays a pivotal role [14]. Nowadays, metabolic syndrome and diabesity are a huge problem, worldwide (specifically for the western world) and the word "huge" is strongly marked by the SARS-Cov2 pandemic that we are living in these days. Massive weight loss in morbidly obese subjects caused marked changes in proinflammatory cytokines and increased the plasmatic levels of adipose tissue metabolism modulators: ghrelin and adiponectin. TNF $\alpha$ system and IL-6 have been implicated in the regulation of energy balance and are considered as potent proinflammatory mediators with apparent effects over many of the hormonal factors produced by the adipose tissue [14]. Several studies have shown increased proinflammatory cytokines in the serum of COVID-19 patients. Also, anti-inflammatory agents for COVID-19 therapy highlight the critical role of inflammation in the progression of COVID-19. Despite the role of inflammatory markers (in general) in monitoring the severity of COVID-19 is still controversial, CRP, PCT, IL-6, and ESR, were positively correlated with the severity of COVID-19 [15]. As mentioned in a previous study [13], the infected people who died were mostly older than 70 years, males, hypertensive, diabetic, and affected by serious comorbidities such as congestive heart failure, chronic obstructive pulmonary diseases, and renal diseases. Males, hypertensive, diabetics, and those with COPD or major CVD showed an approximately doubled risk of death or severe disease. Obesity is also emerging as a significant, independent predictor of severe/lethal disease [13].

The effects of the LCHFFF diet may also activate genes that are critical for the hepatic metabolism of lipids and glucose. However, no hepatic steatosis was detected from the abdominal ultrasound exam. Also, as shown in the table, the LCHFFF diet seems to not have any side effects in the hepatobiliary system: AST, ALT and, GGT levels are perfectly in the range (Table 1). The LCHFFF diet causes an effective and sustained fat loss (BIVA vector, waist-hip ratio: data not shown). This weight loss has been associated with marked improvement in glycemic control, hepatic insulin sensitivity, liver enzymes, and liver histology [16, 17]. Also, it has been demonstrated that each individual differs in response to diet, according to the genetic background [16]. Although obesity and insulin resistance are the most prevalent risk factors for NAFLD pathogenesis, hepatic fat content varies substantially among individuals with equivalent adiposity, indicating that other risk factors may contribute to this condition. Indeed, epidemiological, familial and twin studies provide evidence of a strong heritability of hepatic lipid accumulation [18, 19]. Further research in this area is needed to better understand the complex mechanism between inherited susceptibility, epigenetic signature, gut-liver axis derangement, unhealthy dietary habits, and NAFLD.

Cholesterol gallstones are among the most common gastrointestinal disorders in Western societies. Individuals with gallstones may experience various gastrointestinal symptoms and are also at risk of developing acute or chronic cholecystitis. Cholecystectomy is the most frequently recommended conventional treatment for symptomatic gallstones. Dietary factors that may prevent the development of gallstones include polyunsaturated fat, 
monounsaturated fat, fiber, and caffeine. Consuming a vegetarian diet is also associated with decreased risk [20]. After a month of LCHFFF diet, no variation in biochemical markers of the hepatobiliary system was detected (Table 1).

Several studies in the non-diabetic population showed an increase in the HDL cholesterol and apolipoprotein A-I concentrations with an increased egg intake and minimal effects on LDL cholesterol [21-23]. Even in diabetic people, foods rich in fats and cholesterol like eggs doesn't show any negative effect on blood lipid level in T2D patients [5]. Improvement in blood lipid profiles and adipose tissue can be confounded by the trial being a weight-loss intervention; weight loss itself is not a good predictor of blood lipid levels and adipose tissue status as the NWO syndrome (normalweight obesity syndrome) described.

\section{Conclusion}

A person with metabolic syndrome, T2D (or prediabetes) who consumed an LCHFFF diet (exception for genetic conditions) can decrease the level of triglycerides, improve the levels of total cholesterol, LDL, and HDL, and improve glycemic control.

The decrease in triglycerides with the LCHFFF diet is due to the decrease in the carbohydrates entering the body and increased metabolism. Only fewer carbohydrates have the possibility to be converted into triglycerides.

According to Noakes et al. [4] in the narrative review, a diet low in carbohydrates and rich in fat (LCHF diet $<26 \%$ of total energy intake or $<130 \mathrm{~g} \mathrm{CHO/day)} \mathrm{can} \mathrm{increase} \mathrm{satiety,} \mathrm{allowing} \mathrm{a} \mathrm{lower} \mathrm{energy} \mathrm{intake} \mathrm{without} \mathrm{hunger} \mathrm{and} \mathrm{a}$ specific metabolic advantage and at least as effective as other dietary strategies for reducing body weight. LCHF diets are an effective dietary strategy to improve glycemic control and reduce hyperinsulinemia in T2D. LCHF diets have unique effects on blood lipid concentrations and cardiovascular risk factors, characterised by decreased blood triglycerides, apoB, and saturated fat concentrations, reduced small LDL particle numbers, and increased HDL-C concentrations. The effect on LDL-C concentration is variable.

As previously stated, "In the western countries, preventing conditions like obesity and T2D or other risk factors correlated with metabolism can be one of the ways to increase the general status of health population and make people more resistant towards any unexpected adversity like SARS-Cov-2".

\section{Supplementary Material}

The supplementary material for this article can be found online at: https://seriesscience.com/wpcontent/uploads/2021/09/Supplementary-Material.pdf

\section{References}

1. Wannamethee SG, Shaper AG, Lennon L, et al. Metabolic syndrome vs Framingham risk score for prediction of coronary heart disease, stroke, and type 2 diabetes mellitus. Arch Intern Med. 2005;165(22):2644-650.

2. Koskinen J, Kahonen M, Viikari JS et al. Conventional cardiovascular risk factors and metabolic syndrome in predicting carotid intima-media thickness progression in young adults: the cardiovascular risk in young Finns study. Circulation. 2009;120(3):229-36.

3. Geiker NRW, Larsen ML, Dyerberg J, et al. Eggs do not increase the risk of cardiovascular disease and can be safely consumed. Ugeskr Laeger. 2017;179(20):V11160792.

4. Noakes TD, Windt J. Evidence that supports the prescription of low-carbohydrate high-fat diets: a narrative review. Br J Sports Med. 2017;51(2):133-39.

5. Fuller NR, Caterson ID, Sainsbury A, et al. The effect of a high-egg diet on cardiovascular risk factors in people with type 2 diabetes: the Diabetes and Egg (DIABEGG) study-a 3-mo randomized controlled trial. Am J Clin Nutr 2015;101(4):705-13. 
6. Geiker NRW, Larsen ML, Dyerberg J, et al. Egg consumption, cardiovascular diseases and type 2 diabetes. Eur J Clin Nutr. 2018;72(1):44-56.

7. Sabaté J, Oda K, Ros E. Nut consumption and blood lipid levels: A pooled analysis of 25 intervention trials. Arch Intern Med. 2010;170(9):821-27.

8. Farnetti S, Malandrino N, Luciani D, et al. Food fried in extra-virgin olive oil improves postprandial insulin response in obese, insulin-resistant women. J Med Food . 2011;14(3):316-21.

9. Roden M. How free fatty acids inhibit glucose utilization in human skeletal muscle. News Physiol Sci. 2004;19:92-96.

10. Jaworski K, Sarkadi-Nagy E, Duncan RE, et al. Regulation of triglyceride metabolism. IV. Hormonal regulation of lipolysis in adipose tissue. Am J Physiol Gastrointest Liver Physiol. 2007;293(1):G1-4.

11. Adiels M, Olofsson S-O, Taskinen M-R, et al. Overproduction of very low-density lipoproteins is the hallmark of the dyslipidemia in the metabolic syndrome. Arterioscler Thromb Vasc Biol. 2008;28(7):1225236.

12. Lefebvre $\mathrm{P}$, Cariou B, Lien F, et al. Role of bile acids and bile acid receptors in metabolic regulation. Physiol Rev. 2009;89(1):147-91.

13. Baldini C. A case study: LCHF diet combined with fried food in patient with type 2 diabetes and central obesity reduces need for exogenous insulin injection. Baldini. Res Chronic Dis. 2020;4(3):155.

14. Vendrell J, Broch M, Vilarras N, et al. Resistin, adiponectin, ghrelin, leptin, and proinflammatory cytokines: Relationships in obesity. Obes Res. 2004;12(6):962-71.

15. Zeng F, Huang Y, Guo Y, et al. Association of inflammatory markers with the severity of COVID-19: A meta-analysis. Int J Infect Dis. 2020;96:467-74.

16. Dongiovanni P, Valenti L. A nutrigenomic approach to non-alcoholic fatty liver disease. Int J Mol Sci. 2017;18(7):1534.

17. Meroni M, Longo M, Rustichelli A, et al. Nutrition and genetics in NAFLD: The perfect binomium. Int J Mol Sci. 2020;21(8):2986.

18. Dongiovanni P, Anstee QM, Valenti L. Genetic predisposition in NAFLD and NASH: Impact on severity of liver disease and response to treatment. Curr Pharm Des. 2013;19(29):5219-238.

19. Dongiovanni P, Romeo S, Valenti L. Genetic factors in the pathogenesis of nonalcoholic fatty liver and steatohepatitis. Biomed Res Int. 2015;2015:460190.

20. Gaby AR. Nutritional approaches to prevention and treatment of gallstones. Altern Med Rev. 2009;14(3):258-67.

21. Mutungi G, Ratliff J, Puglisi M, et al. Dietary cholesterol from eggs increases plasma HDL cholesterol in overweight men consuming a carbohydrate-restricted diet. J Nutr. 2008;138(2):272-76.

22. Deng Y, Tang K, Chen R, et al. Effects of Shugan-Jianpi recipe on the expression of the p38 MAPK/NF- $\kappa B$ signaling pathway in the hepatocytes of NAFLD rats. Medicines (Basel). 2018;5(3):106.

23. Knopp RH, Retzlaff B, Fish B, et al. Effects of insulin resistance and obesity on lipoproteins and sensitivity to egg feeding. Arterioscler Thromb Vasc Biol. 2003;23(8):1437-443. 